\title{
Identification of key genes in osteoarthritis using bioinformatics, principal component analysis and meta-analysis
}

\author{
XIANGXIANG SUN, HONGHAO DUAN, LIN XIAO, SHUXIN YAO, QIANG HE, \\ XINLIN CHEN, WEIJIE ZHANG and JIANBING MA
}

\begin{abstract}
Department of Knee Surgery, Hong-Hui Hospital, Xi'an Jiaotong University College of Medicine, Xi'an, Shaanxi 710054, P.R. China
\end{abstract}

Received April 20, 2018; Accepted November 22, 2018

DOI: $10.3892 /$ etm.2020.9450

\begin{abstract}
The present study aimed to identify key genes involved in osteoarthritis (OA). Based on a bioinformatics analysis of five gene expression profiling datasets (GSE55457, GSE55235, GSE82107, GSE12021 and GSE1919), differentially expressed genes (DEGs) in OA were identified. Subsequently, a protein-protein interaction (PPI) network was constructed and its topological structure was analyzed. In addition, key genes in OA were identified following a principal component analysis (PCA) based on the DEGs in the PPI network. Finally, the functions and pathways enriched by these key genes were also analyzed. The PPI network consisted of 241 nodes and 576 interactives, including a total of 171 upregulated DEGs [e.g., aspartylglucosaminidase (AGA), CD58 and CD86] and a total of 70 downregulated DEGs (e.g., acetyl-CoA carboxylase $\beta$ and dihydropyrimidine dehydrogenase). The PPI network complied with an attribute of scale-free small-world network. After PCA, 47 key genes were identified, including $\beta$-1,4-galactosyltransferase-1 (B4GALT1), AGA, CD58, CD86, ezrin, and eukaryotic translation initiation factor $4 \gamma 1$ (EIF4G1). Subsequently, the 47 key genes were identified to be enriched in 13 Gene Ontology (GO) terms and 2 Kyoto Encyclopedia of Genes and Genomes pathways, with the GO terms involving B4GALT1 including positive regulation of developmental processes,
\end{abstract}

Correspondence to: Dr Jianbing Ma, Department of Knee Surgery, Hong-Hui Hospital, Xi'an Jiaotong University College of Medicine, 555 Eastern Youyi Road, Xi'an, Shaanxi 710054, P.R. China E-mail: jianbingmap22@sina.com

Abbreviations: OA, osteoarthritis; DEGs, differentially expressed genes; PPI, protein-protein interaction; PCA, principal component analysis; MMP, matrix metalloproteinase; IL-6, interleukin 6; GEO, Gene Expression Omnibus; PC, principal component; GO, Gene Ontology; KEGG, Kyoto Encyclopedia of Genes and Genomes; RT-qPCR, reverse transcription-quantitative polymerase chain reaction; OR, odds ratio

Key words: osteoarthritis, genomic meta-analysis, principal component analysis, network protein amino acid terminal glycosylation and protein amino acid terminal $\mathrm{N}$-glycosylation. In addition, B4GALT1 and EIF4G1 were confirmed to be downregulated in OA samples compared with healthy controls, but only EIF4G1 was determined to be significantly downregulated in OA samples, as determined via a meta-analysis of the 5 abovementioned datasets. In conclusion, B4GALT1 and EIF4G1 were indicated to have significant roles in OA, and B4GALT1 may be involved in positive regulation of developmental processes, protein amino acid terminal glycosylation and protein amino acid terminal $\mathrm{N}$-glycosylation. The present study may enhance the current understanding of the molecular mechanisms of $\mathrm{OA}$ and provide novel therapeutic targets.

\section{Introduction}

Osteoarthritis (OA), a degenerative joint disease, has various characteristic symptoms, including as joint pain, swelling, stiffness and a limited range of motion $(1,2)$. As the most common form of arthritis, OA affects the normal daily activity of patients and their quality of life (3). An estimated $3.3 \%$ of the global population suffer from OA (4) and $30 \%$ of individuals affected are aged $>60$ years (3). Although OA is closely associated with age, the underlying mechanisms of the disease remain to be fully elucidated.

In the past, OA was regarded as a disease that only comprises cartilage degradation (5). However, it is a complex process caused by diverse factors, including inflammatory proteins (6), matrix-degrading enzymes (7), synovitis (8) and genetic factors (9). For instance, chondrocytes may be activated by mechanical pressure, leading to an increase in the secretion of proteases, cytokines and inflammatory mediators, as well as the degradation of collagen protein in obese patients (10). In addition, the levels of collagen, matrix metalloproteinases (MMPs) and cytokines, including interleukin 6 (IL-6) and tumor necrosis factor, were reported to increase after chondrocyte activation (11-13). By contrast, tissue inhibitor of MMPs and suppressive cytokines, including IL-4, IL-10 and IL-13, were significantly decreased after chondrocyte activation in OA $(14,15)$. Thus, the progression of OA is associated with an increase of inflammatory cytokines and reduced suppressive cytokines. In addition, studies have focused on genetic factors in $\mathrm{OA}$ in recent years. A reduction 
in the expression levels of growth differentiation factor 5 due to the single nucleotide polymorphism rs143383 was reported to be associated with OA (16). Furthermore, urinary collagen type II C-telopeptide was identified as a prognostic marker in knee and hip OA (17). Overexpression of tuftelin 1 in ATDC5 cells inhibited chondrogenic differentiation, a step in the pathogenesis of OA, by increasing calcification (18). Therefore, it is important to study the mechanisms underlying the OA-associated processes.

The present study aimed to identify key genes in OA through analyzing five public gene expression profiling datasets by using a genomic meta-analysis. In the present study, the differentially expressed genes (DEGs) in OA vs. controls were identified using bioinformatics. Subsequently, a protein-protein interaction (PPI) network for these DEGs was generated and analyzed. In addition, the key genes were identified using principal component analysis (PCA) of the DEGs in the PPI network. The functions and pathways of these key genes were also elucidated by using gene ontology (GO) and Kyoto Encyclopedia of Genes and Genomes (KEGG) enrichment analyses. Finally, the differential expression of two key genes identified was verified using a meta-analysis of the expression data from the different data-sets and by gene expression analysis in a rat model of OA.

\section{Materials and methods}

Data acquisition and pre-processing. The key words 'osteoarthritis' and 'Homo sapiens' were used to search for all relevant public expression profiling data in the Gene Expression Omnibus database (GEO; http://www.ncbi. nlm.nih.gov/geo/) deposited until June 10, 2017. A total of five datasets generated with the Affymetrix platforms were downloaded (Table I). The data in CEL files were pre-processed with the oligo package in $\mathrm{R}$ (19), including data format transformation, supplementation of missing values using the median method, background correction using the MicroArray Suite 5.0 (MAS5, Affymetrix; Thermo Fisher Scientific, Inc., Waltham, MA, USA) and quantiles normalization $(20,21)$.

Identification of DEGs using genomic meta-analysis. The major purpose of the present meta-analysis was to integrate the results of multiple datasets and screen out significant DEGs. First of all, the MetaQC package in R (http://cran.r-project. org/) was used to perform a quality control (QC) of the datasets, comprising internal QC, external QC, accuracy QC and consistency QC (22). In addition, PCA biplots and standardized mean rank (SMR) were used for assistance in decision making regarding the QC results and for screening the appropriate dataset (23). Subsequently, the MetaDE package was used to identify the DEGs using meta-analysis based on the reliable datasets according to QC (24). Statistical parameters of heterogeneity (e.g., $\mathrm{I}^{2}$ and Qpval) were obtained by a heterogeneity test for each gene assessed using different platforms with the MetaDE.ES method. When $\mathrm{I}^{2}=0$ and Qpval >0.05, the gene expression was considered homogeneous without bias. The differential expression of genes in the integrated dataset was detected. Only those with a false discovery rate (FDR) of $<0.05$ were considered significantly different. Finally, genes with $\mathrm{I}^{2}=0$, Qpval $>0.05, \log _{2}$ fold change $>0.58$ and FDR $<0.05$ were identified as DEGs. Furthermore, the hierarchical clustering heatmap for DEGs in the five datasets was obtained using the heatmap.sig. genes function in MetaDE package (https://cran.r-project. org/web/packages/MetaDE/).

Construction of the PPI network. The Search Tool for the Retrieval of Interacting Genes/Proteins (STRING) database (version 10.5, http://string-db.org/) was used to determine the interactive association among DEGs, and a PPI network including the DEGs and their interactions was then constructed (25). A combined score (including Neighborhood in the Genome score, Gene Fusions score, Cooccurence Across Genomes score, Co-Expression score, Experimental/Biochemical Data score, Association in Curated Databases score, and Co-Mentioned in PubMed Abstracts score) $>0.4$ under default parameters was set as a threshold value. Subsequently, the PPI network was visualized using Cytoscape software (version 3.2.0, http://www.cytoscape. $\operatorname{org} /)$.

Topological analysis of the PPI network. Deletion of the number of links per node (node degree) in the PPI network of Saccharomyces cerevisiae and Escherichia coli indicated that the degree of connectedness of a protein has an important role (26). Hub nodes were identified when the degree of interactions was $>15$ and the average shortest path length was $<3.5$.

Identification of the key genes using PCA. PCA is a feature dimension reduction procedure that is able to identify new variables or PCs (27). A set of possibly correlated variables was converted into a set of values of linearly uncorrelated variables called PCs using orthogonal transformation through PCA. If the new variable has the largest possible variance, it is regarded as the first PC. After extraction of the first PC, each succeeding component in turn has the highest variance. Thus, the variance and information content of each PC are diminishing consecutively $(\mathrm{PC} 1>\mathrm{PC} 2 \ldots>\mathrm{PCn})$. The PCA of DEGs in the PPI network was performed with the psych package in $\mathrm{R}$ software. The key genes in each dataset were thereby identified.

GO term and KEGG pathway enrichment analysis for the key genes. The Database for Annotation, Visualization and Integrated Discovery (DAVID; version 6.7; https://david. ncifcrf.gov/), a bioinformatics web tool for the functional annotation analysis of high-throughput gene lists, was used to analyze GO terms and KEGG pathways enriched by the key genes $(28,29)$. The significance of the terms/pathways $(\mathrm{P}<0.05)$ was identified according to their hypergeometric distribution.

Signature analysis of the key genes. The Meta package in $\mathrm{R}$ software was used to analyze the expression features of the key genes. Forest plots for the expression of the key genes between $\mathrm{OA}$ samples and control samples were obtained via the fixed-effect model with $\mathrm{I}^{2}=0$. 
Table I. Characteristics of five datasets from the Affymetrix platforms of Gene Expression Omnibus.

\begin{tabular}{lccc}
\hline Accession no. & Number of OA samples & Number of normal samples & Platform \\
\hline GSE55235 & 10 & 10 & Affymetrix Human Genome GPL96 Array \\
GSE55457 & 10 & 10 & Affymetrix Human Genome GPL96 Array \\
GSE1919 & 5 & 5 & Affymetrix Human Genome GPL96 Array \\
GSE12021 & 10 & 9 & Affymetrix Human Genome GPL96 Array \\
GSE82107 & 10 & 7 & Affymetrix Human Genome GPL570 Array
\end{tabular}

OA, osteoarthritis.

Table II. Sequences of primers used for polymerase chain reaction.

\begin{tabular}{ll}
\hline Primer & \multicolumn{1}{c}{ Sequence (5'-3') } \\
\hline GAPDH-F & AGACAGCCGCATCTTCTTGT \\
GAPDH-R & CTTGCCGTGGGTAGAGTCAT \\
B4GALT1-F & TCGGGTTTAGCCTGCCTTAC \\
B4GALT1-R & GATCATGCGACACCTGCCTA \\
EIF4G1-F & GACACAAATGAACACGCCTTCT \\
EIF4G1-R & CCAGCAGGGTAGACATGGGG \\
\hline
\end{tabular}

$\mathrm{F}$, forward primer; $\mathrm{R}$, reverse primer; B4GALT1, $\beta$-1,4-galactosyltransferase-1; EIF4G1, eukaryotic translation initiation factor $4 \gamma 1$.

Animal model of OA. Male Sprague Dawley (SD) rats (weight, $220 \pm 25 \mathrm{~g}$; age, 8 weeks) were used to establish an animal model of OA and were randomly divided into the normal control and model groups (30 rats per group). The SD rats were purchased from SLAC Laboratory Animal Co., Ltd. (Shanghai, China) and kept at $20 \pm 2{ }^{\circ} \mathrm{C}$ and relative humidity of $55 \pm 5 \%$ with a 12-h light/dark cycle under specific pathogen-free conditions. After acclimatization for 3 days and on the fourth day, rats in the control group were fed ad libitum water and food without any other treatment. The left and right knee joints of the rats in the model group were subjected to anterior cruciate ligament transection and immobilized thereafter (30). At 8 weeks post-surgery, those rats in which the OA model was successfully established and rats in normal group were anesthetized by intraperitoneal injection of $3 \%$ chloral hydrate $(300 \mathrm{mg} / \mathrm{kg})$ prior to decapitation, and their joints and synovial tissues were harvested.

Verification of DEGs in OA by reverse transcription-quantitative polymerase chain reaction ( $R T-q P C R)$. To confirm the results of the meta-analysis, the expression levels of $\beta$-1,4-galactosyltransferase-1 (B4GALT1) and eukaryotic translation initiation factor $4 \gamma 1$ (EIF4G1) were detected in the synovial tissues of the rat model of OA using RT-qPCR. Total RNA was extracted from synovial tissues of the rats using the RNAiso Plus reagent (cat. no. 9109; Takara Bio, Inc., Otsu, Japan) according to the manufacturer's protocol under low temperature. Subsequently, the first-strand complementary DNA was prepared from synovial tissue RNA using the PrimeScript ${ }^{\mathrm{TM}}$ RT Master Mix (cat. no. RR036A; Takara Bio, Inc.) according to the manufacturer's protocol. GAPDH was detected as an endogenous control. The primers used for B4GALT1, EIF4G1 and GAPDH were based on the rat sequences (Table II). The thermocycling protocol for PCR was as follows: $50^{\circ} \mathrm{C}$ for $3 \mathrm{~min}, 95^{\circ} \mathrm{C}$ for $3 \mathrm{~min}$, followed by 40 cycles of $95^{\circ} \mathrm{C}$ for $10 \mathrm{sec}$ and $60^{\circ} \mathrm{C}$ for $30 \mathrm{sec}$. The relative amounts of the mRNAs were obtained by using the Relative Expression Software Tool (REST, Version 2.0.7, Corbett Life Science; Qiagen, Inc., Valencia, CA, USA), and relative gene expression was calculated using the $2^{-\Delta \Delta \mathrm{Cq}}$ method (31).

Statistical analysis. All experiments were performed three times, and the results are expressed as the mean \pm standard error of the mean. Differences in gene expression between the OA and normal control groups were compared using an unpaired two-sided Student's t-test with SPSS 22.0 (IBM Corp., Armonk, NY, USA). $\mathrm{P}<0.05$ was considered to indicate a statistically significant difference.

\section{Results}

Quality assessment of the five datasets. The different QC measures and SMR scores are presented in Table III. The SMR scores in the five datasets were similar, indicating a high homogeneity and low bias among the five datasets. Together with the PCA biplots, the first two PCs covered up to $88.41 \%$ of variance (Fig. 1). In addition, the five datasets met the criteria regarding the selected parameters and exhibited no marked differences. After comprehensively considering multiple parameters, the five datasets were identified as being apt for simultaneous inclusion in the present meta-analysis.

DEGs in OA from the five datasets. In total, 1,007 DEGs between $\mathrm{OA}$ and control samples were identified in the five datasets. In addition, the heatmap indicated that it these DEGs were distinguishable between OA and control samples (Fig. 2).

Topological structure of the PPI network. The PPI network contained 241 nodes, including 171 upregulated DEGs [e.g., cyclin-dependent kinase 1 (CDK1), aspartylglucosaminidase (AGA), CD58 and CD86] and 70 downregulated DEGs 
Table III. Different QC measures and SMR scores.

\begin{tabular}{lccccccc}
\hline Dataset & IQC & EQC & CQCg & CQCp & AQCg & AQCp & SMR \\
\hline GSE55457 & 4.91 & 4.78 & 93.87 & 148.67 & 153.83 & 56.44 & 2.42 \\
GSE55235 & 4.56 & 4.22 & 68.15 & 146.58 & 106.19 & 29.43 & 2.83 \\
GSE82107 & 4.38 & 3.16 & 64.14 & 146.51 & 26.46 & 96.74 & 2.42 \\
GSE12021 & 4.81 & 3.23 & 59.25 & 171.49 & 25.5 & 84.37 & 2.86 \\
GSE1919 & 5.12 & 5 & 52.41 & 101.36 & 184.06 & 39.3 & 1.57 \\
\hline
\end{tabular}

QC, quality control; SMR, standardized mean rank; IQC, internal QC; EQC, external QC; CQCg, consistency QC of differentially expressed gene ranking; CQCp, consistency QC of enriched pathway ranking; AQCg, accuracy QC of gene detection; AQCp, accuracy QC of enriched pathway detection.

[e.g., acetyl-CoA carboxylase $\beta$ (ACACB) and dihydropyrimidine dehydrogenase (DPYD)] and 576 interactive associations (Fig. 3A). The PPI network complied with an attribute of scale-free small-world network (Fig. 3B). The node degree function followed the power-law distribution with an algorithm of $y=47.82 \mathrm{~d}^{-2.46}$, where $\mathrm{d}$ indicates the degree of connectivity (the number of links in the PPI). In addition, the most frequent path length of the nodes was 4 , which was also in accordance with scale-free networks with a small-world effect $(32,33)$ (Fig. 3C).

Key genes identified by PCA. By performing a PCA, 20, 20, 17, 19 and 10 PCs were obtained in the datasets GSE55457, GSE55235, GSE82107, GSE12021 and GSE1919, respectively. As presented in Table IV, the first three PCs covered $>80 \%$ of variance. Thus, the three PCs contained more important information of the original variable (gene expression values). This was further verified in three-dimensional graphs (Fig. 4). PC1, PC2 and PC3 were able to distinguish the OA samples and control samples in each dataset. If the absolute value of a variable coefficient in a PC was large, the variable had an important role in the PC. Therefore, the genes with an absolute value of the coefficient of $>0.1$ were defined as the key genes. In the present study, 47 key genes were identified, including B4GALT1, AGA, CD58, CD86, ezrin, adaptor-related protein complex 3 subunit $\delta 1$ and EIF4G1. Subsequently, the 47 key genes were identified to be enriched in $13 \mathrm{GO}$ terms in the category biological process and 2 KEGG pathways (Table V). For instance, B4GALT1 was mainly involved in the positive regulation of developmental processes, protein amino acid terminal glycosylation and protein amino acid terminal $\mathrm{N}$-glycosylation. In addition, the forest plots of the expression levels of the key genes in $\mathrm{OA}$ and control samples indicated that EIF4G1 was significantly downregulated in OA samples [odds ratio $(\mathrm{OR})=0.20$; 95\% confidence interval (CI): 0.07-0.57; Fig. 5A]. However, B4GALT1 expression was not significantly different between $\mathrm{OA}$ and control samples $(\mathrm{OR}=0.43$; 95\% CI, 0.17-1.03; Fig. 5A). Furthermore, rat tissues from the model group exhibited a success rate of $76.7 \%$ (23/30). RT-qPCR analysis of the synovial tissues of rat model of OA confirmed that the expression levels of B4GALT1 $(\mathrm{P}=0.0002)$ and EIF4G1 $(\mathrm{P}=0.0495)$ were significantly decreased in $\mathrm{OA}$ vs. control tissues (Fig. 5B).
Table IV. Top 3 PCs (\%) in the five datasets according to PCA.

\begin{tabular}{llll}
\hline Dataset & PC1 & PC2 & PC3 \\
\hline GSE55457 & 39.93 & 21.72 & 21.43 \\
GSE55235 & 37.99 & 30.49 & 18.36 \\
GSE82107 & 31.17 & 28.48 & 26.14 \\
GSE12021 & 30.76 & 28.14 & 21.54 \\
GSE1919 & 37.33 & 22.08 & 20.67
\end{tabular}

PCA, principal component analysis.

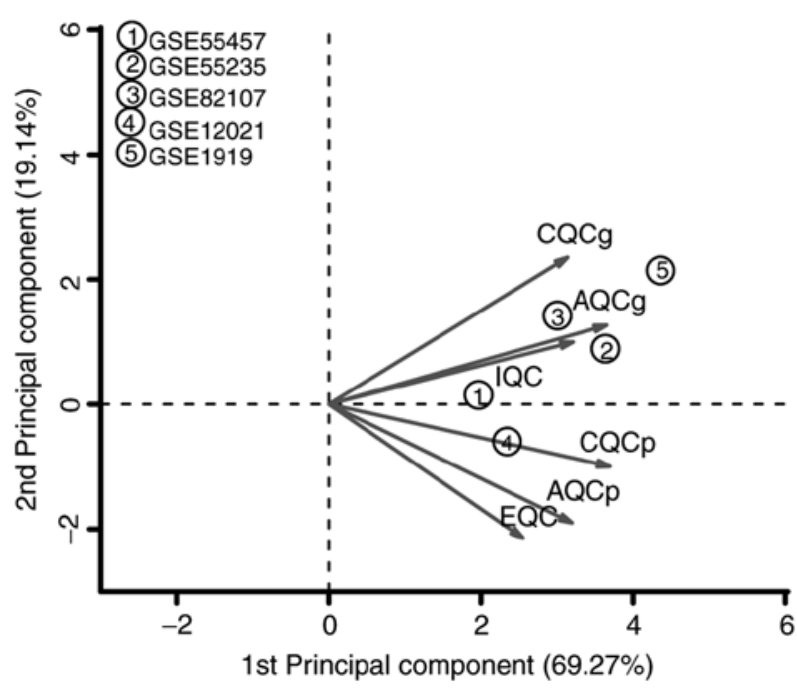

Figure 1. PCA biplots of QC measures for the five datasets included in the present meta-analysis. The abscissa indicates the first $\mathrm{PC}$ and the ordinate indicates the second PC in the PCA. QC, quality control; IQC, internal QC; EQC, external QC; CQCg, consistency QC of differentially expressed gene ranking; $\mathrm{CQCp}$, consistency $\mathrm{QC}$ of enriched pathway ranking, $\mathrm{AQCg}$, accuracy QC of gene detection; AQCp, accuracy QC of enriched pathway detection, PCA, principal component analysis.

\section{Discussion}

In the present study, a total of 1,007 DEGs were identified between $\mathrm{OA}$ and control samples in five gene expression datasets using a bioinformatics analysis. Subsequently, a PPI network with a scale-free small world topology was 
Table V. GO terms in the category biological process and KEGG pathways enriched by the 47 key genes in osteoarthritis.

A, GO terms

\begin{tabular}{|c|c|c|c|}
\hline Term & Count & P-value & Genes \\
\hline $\begin{array}{l}\text { GO:0051094-positive regulation of developmental } \\
\text { process }\end{array}$ & 6 & 0.00179 & $\begin{array}{l}\text { B4GALT1, ACVR2B, CD86, BTC, } \\
\text { AP3D1, ADAM9 }\end{array}$ \\
\hline GO:0006494-protein amino acid terminal glycosylation & 2 & 0.00635 & B4GALT1, DDOST \\
\hline $\begin{array}{l}\text { GO:0006496-protein amino acid terminal } \\
\text { N-glycosylation }\end{array}$ & 2 & 0.00635 & B4GALT1, DDOST \\
\hline GO:0030855-epithelial cell differentiation & 4 & 0.00933 & B4GALT1, EZR, FZD1, ADAM9 \\
\hline GO:0016337-cell-cell adhesion & 5 & 0.01121 & EZR, COL13A1, CD58, DSC2, ADAM9 \\
\hline GO:0007155-cell adhesion & 7 & 0.02238 & $\begin{array}{l}\text { B4GALT1, EZR, COL13A1, CD58, } \\
\text { DSC2, ECM2, ADAM9 }\end{array}$ \\
\hline GO:0022610-biological adhesion & 7 & 0.02252 & $\begin{array}{l}\text { B4GALT1, EZR, COL13A1, CD58, } \\
\text { DSC2, ECM2, ADAM9 }\end{array}$ \\
\hline GO:0009100-glycoprotein metabolic process & 4 & 0.02614 & B4GALT1, AGA, EXT1, DDOST \\
\hline GO:0007160-cell-matrix adhesion & 3 & 0.03246 & COL13A1, ECM2, ADAM9 \\
\hline GO:0060429-epithelium development & 4 & 0.03519 & B4GALT1, EZR, FZD1, ADAM9 \\
\hline GO:0045597-positive regulation of cell differentiation & 4 & 0.03597 & ACVR2B, CD86, BTC, AP3D1 \\
\hline GO:0031589-cell-substrate adhesion & 3 & 0.03870 & COL13A1, ECM2, ADAM9 \\
\hline GO:0045321-leukocyte activation & 4 & 0.04130 & CD86, DOCK2, DDOST, ADAM9 \\
\hline
\end{tabular}

B, KEGG pathways

\begin{tabular}{lccl}
\hline Pathway ID & Count & P-value & Genes \\
\hline hsa04142: Lysosome & 6 & $\begin{array}{l}0.00038 \\
\text { hsa05323: Rheumatoid arthritis }\end{array}$ & $\begin{array}{l}\text { AGA, CTSK, AP1S2, CTSO, GALC, AP3D1 } \\
\text { CD86, CTSK }\end{array}$ \\
\hline
\end{tabular}

KEGG, Kyoto Encyclopedia of Genes and Genomes; GO, gene ontology; hsa, Homo sapiens. B4GALT1, $\beta$-1,4-galactosyltransferase-1; ACVR2B, activin A receptor type 2B; CD86, CD86 molecule; BTC, betacellulin; AP3D1, adaptor related protein complex 3 subunit delta 1; ADAM9, ADAM metallopeptidase domain 9; DDOST, dolichyl-diphosphooligosaccharide-protein glycosyltransferase non-catalytic subunit; EZR, ezrin; FZD1, frizzled class receptor 1; COL13A1, collagen type XIII alpha 1 chain; CD58, CD58 molecule; DSC2, desmocollin 2; ECM2, extracellular matrix protein 2; AGA, aspartylglucosaminidase; EXT1, exostosin glycosyltransferase 1; ACVR2B, activin A receptor type 2B; DOCK, DOCK C2 domain containing; CTSK, cathepsin K; AP1S2, adaptor related protein complex 1 subunit sigma 2; CTSO, cathepsin O; GALC, galactosylceramidase.

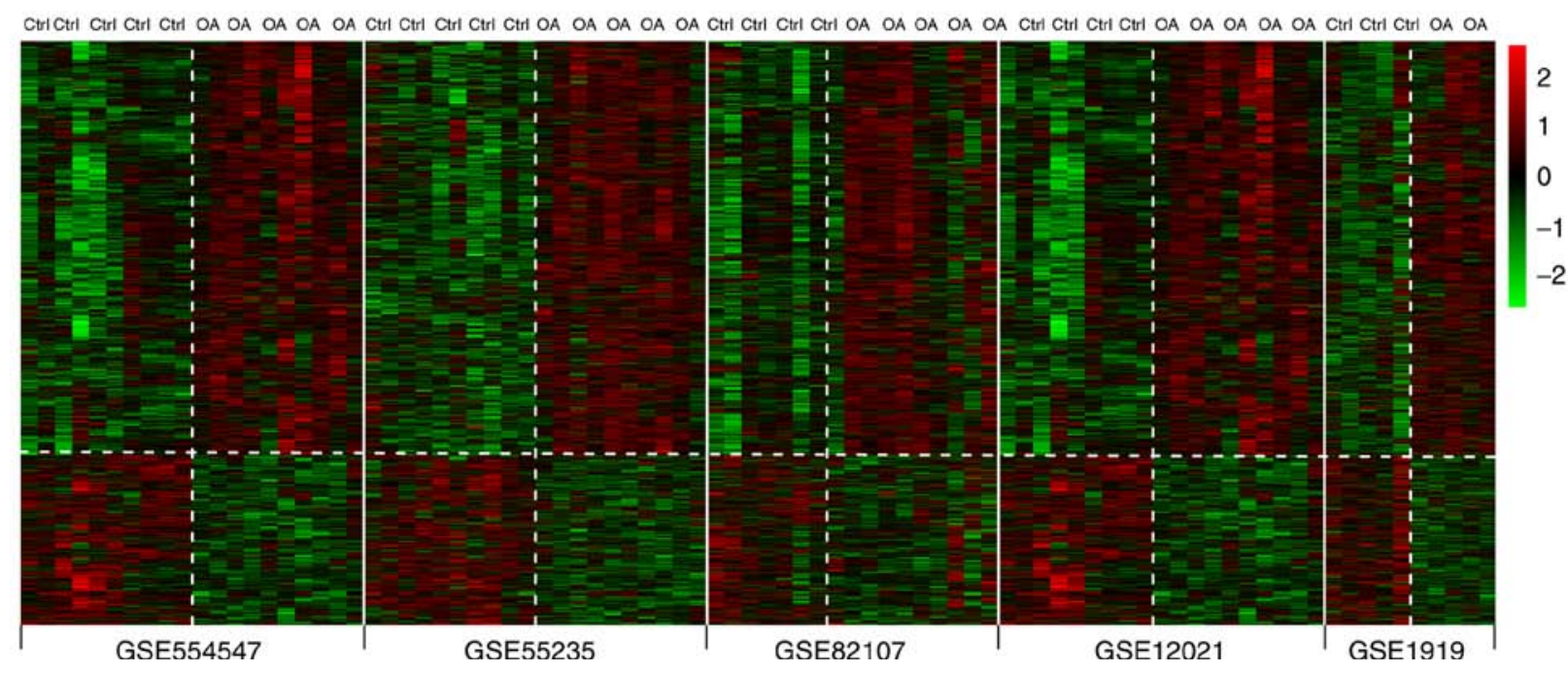

Figure 2. Hierarchical cluster heatmap for the differentially expressed genes from the five datasets (GSE55457, GSE55235, GSE82107, GSE12021 and GSE1919). OA, osteoarthritis; Ctrl, control. 


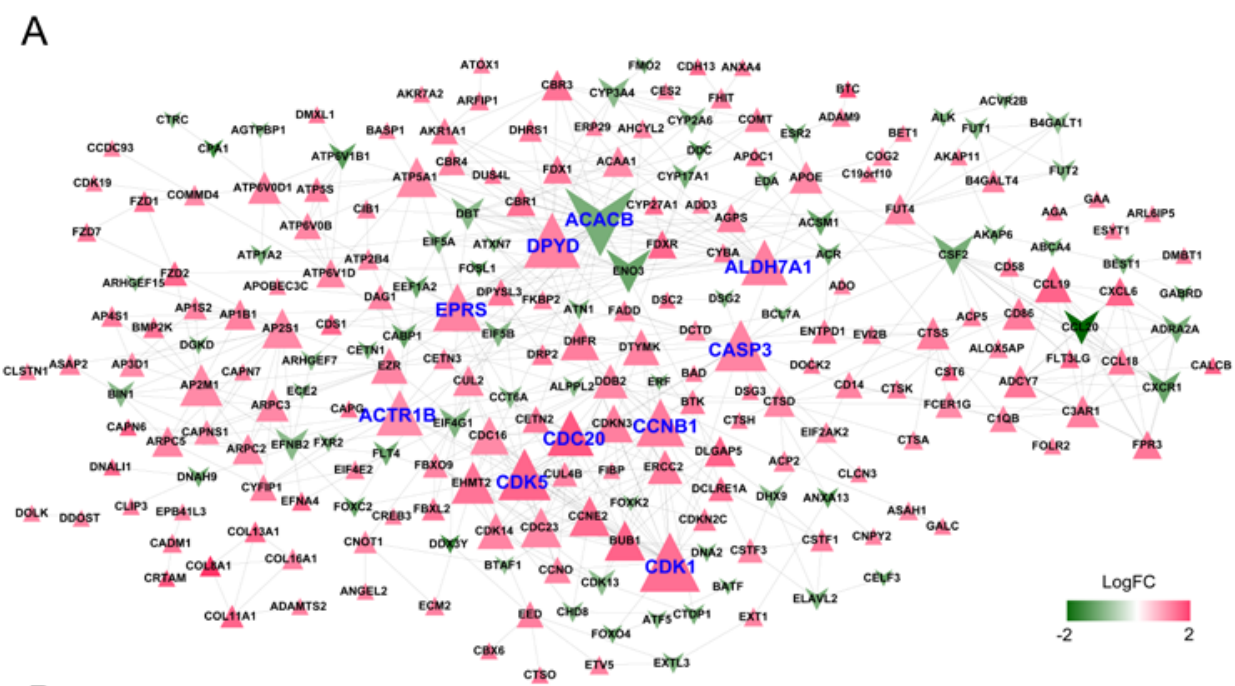

B

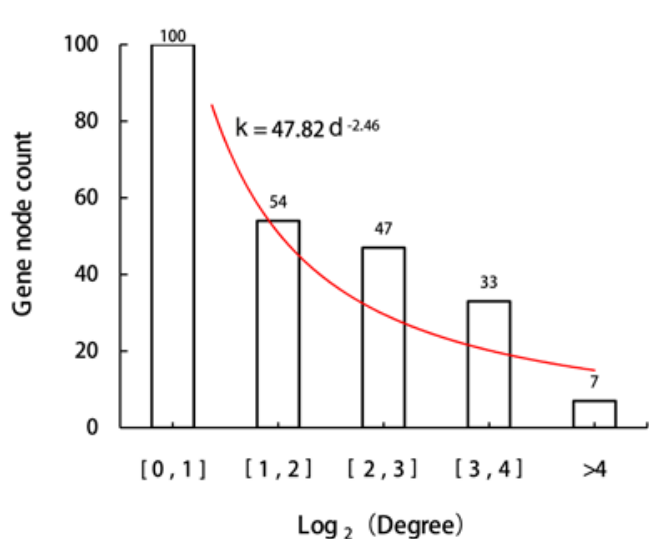

C

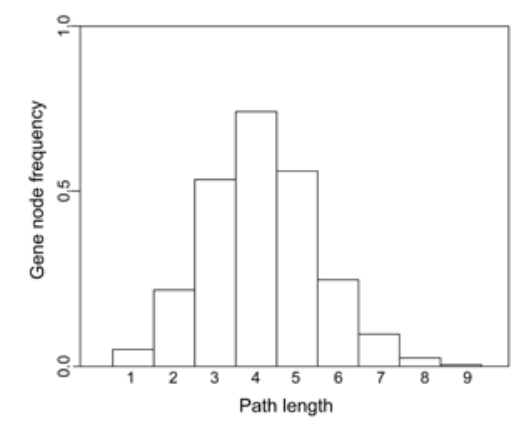

Figure 3. PPI network with a scale-free small world effect based on DEGs. (A) PPI network. Red indicates upregulated DEGs and green indicates downregulated DEGs. The color intensity indicates the $\log \mathrm{FC}$. The node size is proportional to its corresponding degree value and the names of hub genes are marked in dark blue. (B) The distribution of the degree of interaction of the nodes in the network. Scale-free feature means the degree of a small number of nodes are large, while that of most nodes are small. Small world effect means most nodes are not neighbors of one another, while they can be reached from any other nodes by a small number of steps. The x-axis displays the log2-transformed degree of interaction, while the y-axis indicates the numbers of the nodes. (C) Distribution of the path length of nodes in the network. FC, fold change; PPI, protein-protein interaction; DEG, differentially expressed gene.

constructed from these DEGs, including 241 nodes and 576 interactive associations. Of the nodes in the PPI, 171 were upregulated DEGs (e.g., CDK1, AGA, CD58 and CD86) and 70 were downregulated DEGs (e.g., ACACB and DPYD). After a PCA analysis, 47 key genes were identified, including B4GALT1 and EIF4G1, which were confirmed to be significantly downregulated via RT-qPCR analysis of synovial tissues of OA model rats. However, only EIF4G1 expression was determined to be significantly downregulated in OA samples by a meta-analysis of the 5 datasets. In addition, B4GALT1 was mainly enriched in GO terms including positive regulation of developmental processes, protein amino acid terminal glycosylation and protein amino acid terminal $\mathrm{N}$-glycosylation.

B4GALT1 is a member of the family of B4GALT genes and is unique among them, as it encodes an enzyme that participates in glycoconjugate and lactose biosynthesis by transferring uridine diphosphate-bound galactose to terminal $\mathrm{N}$-acetylglucosamines in carbohydrate chains $(34,35)$. Numerous studies have confirmed that B4GALT1 has a key role in inflammatory processes associated with numerous diseases, including $\mathrm{OA}$ and rheumatic arthritis (RA). A previous study indicated that the expression of B4GALT1 was induced in the cartilage and synovial tissue of OA patients when compared with healthy controls and may be an important a key inflammatory mediator in OA (36). By contrast, the expression of B4GALT1 was identified to be downregulated in OA samples in the present study. However, another recent study identified that phosphocitrate inhibited OA in Hartley guinea pigs through upregulating certain genes of the transforming growth factor- $\beta$ receptor signaling pathway, including B4GALT1, and downregulating other genes associated with proliferation and apoptosis (37). This indicates that the expression of B4GALT1 may be reduced in OA, and that its direct or indirect upregulation may represent a therapeutic strategy for OA. In addition, B4GALT1 was mainly enriched in biological processes including positive regulation of developmental processes, protein amino acid terminal glycosylation and protein amino acid terminal N-glycosylation. This is due to B4GALT1, a type II membrane protein, having a hydrophobic N-terminal region, which is able to direct the protein to the Golgi apparatus. Overall, B4GALT1 may be 
A Principal component analysis for GSE55457

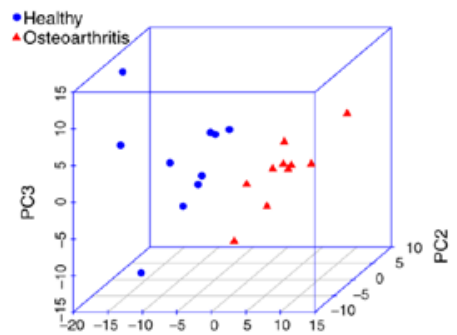

$\mathrm{PC} 1$
B Principal component analysis for GSE55235

- Healthy

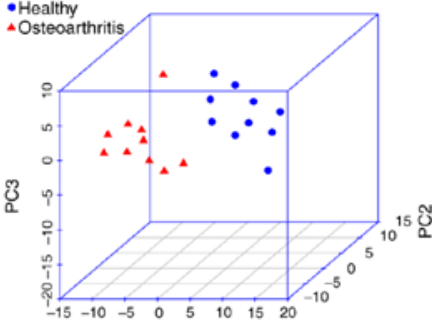

PC1
C Principal component analysis for GSE82107

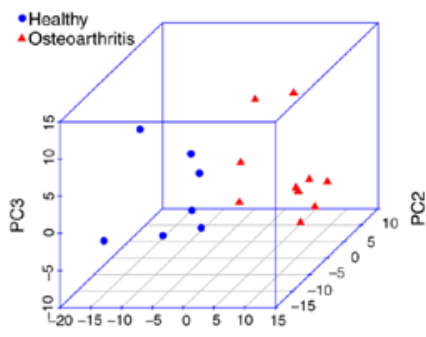

$\mathrm{PC} 1$

D Principal component analysis for GSE12021

E Principal component analysis for GSE1919 - Healthy
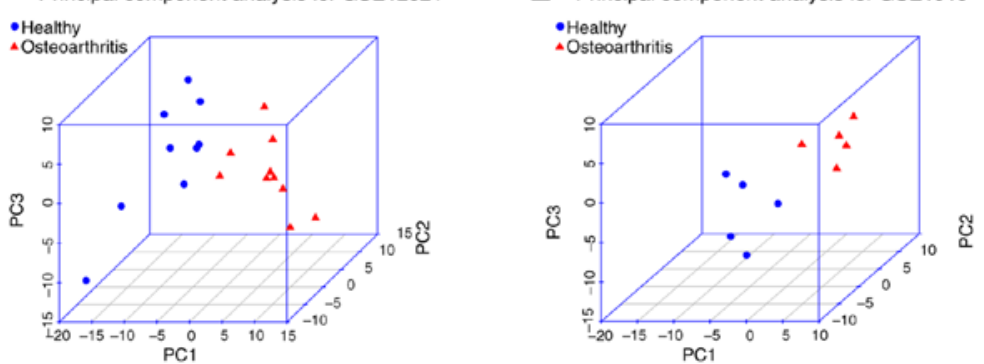

Figure 4. Three-dimensional graph of sample distribution based on three principal components in the five datasets. (A) GSE55457, (B) GSE55235, (C) GSE82107, (D) GSE12021 and (E) GSE1919. Blue dots indicate the control samples and red triangles indicate osteoarthritis samples. PC, principal component.

A

\begin{tabular}{llll}
\multicolumn{1}{c}{ ID } & \multicolumn{1}{c}{ Sample } & Pt & \multicolumn{1}{c}{ PMID } \\
GSE55457 & 20 & GPL96 & 24690414 \\
GSE55235 & 20 & GPL96 & 24690414 \\
GSE82107 & 17 & GPL570 & 27870898 \\
GSE12021 & 19 & GPL96 & 18721452 \\
GSE1919 & 10 & GPL91 & 20858714
\end{tabular}

Fixed effect model : $1^{2}=0$

\begin{tabular}{crcc}
\multicolumn{4}{c}{ B4GALT1 } \\
Osteoarthritis & \multicolumn{2}{c}{ Control } \\
Over-exp & Total & Over-exp Total \\
4 & 10 & 8 & 10 \\
6 & 10 & 7 & 10 \\
7 & 10 & 5 & 7 \\
4 & 10 & 8 & 9 \\
3 & 5 & 2 & 5 \\
& 45 & & 41
\end{tabular}

41

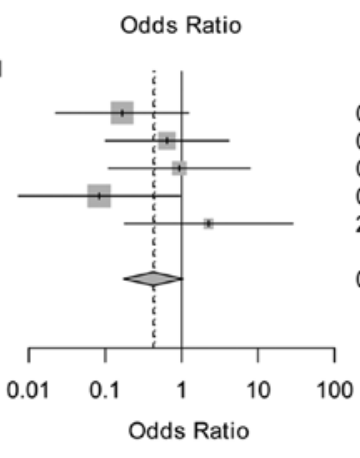

OR $\quad 95 \%-\mathrm{Cl}$

$0.17[0.02 ; 1.23]$

$0.64[0.10 ; 4.10]$

$0.93[0.11 ; 7.82]$

$0.08[0.01 ; 0.95]$

$2.25[0.18 ; 28.25]$

$0.43[0.17 ; 1.03]$

EIF4G1

Osteoarthritis Control

Over-exp Total Over-exp Total

\begin{tabular}{ccll} 
ID & $\begin{array}{c}\text { Sample } \\
(n)\end{array}$ & Pt & \multicolumn{1}{c}{ PMID } \\
& & \\
GSE55457 & 20 & GPL96 & 24690414 \\
GSE55235 & 20 & GPL96 & 24690414 \\
GSE82107 & 17 & GPL570 & 27870898 \\
GSE12021 & 19 & GPL96 & 18721452 \\
GSE1919 & 10 & GPL91 & 20858714
\end{tabular}

Fixed effect model : $\left.\right|^{2}=0$

7
5
5
5
3

10

10
10

10
9
6
6
5

45
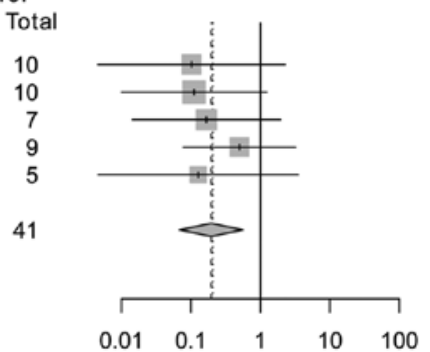

OR $\quad 95 \%-\mathrm{Cl}$

$0.10[0.00 ; 2.28]$

$0.11[0.01 ; 1.24]$

$0.17[0.01 ; 1.94]$

$0.50[0.08 ; 3.21]$

$0.13[0.00 ; 3.52]$

$0.20[0.07 ; 0.57]$
B

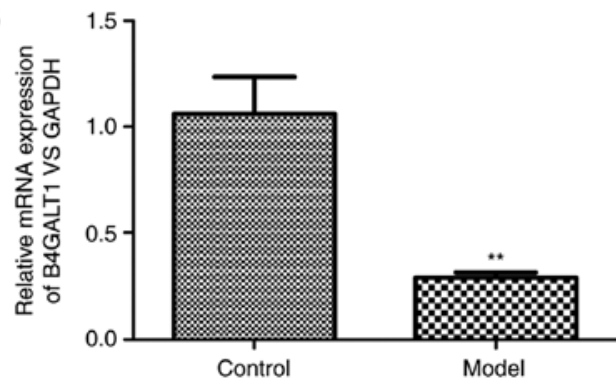

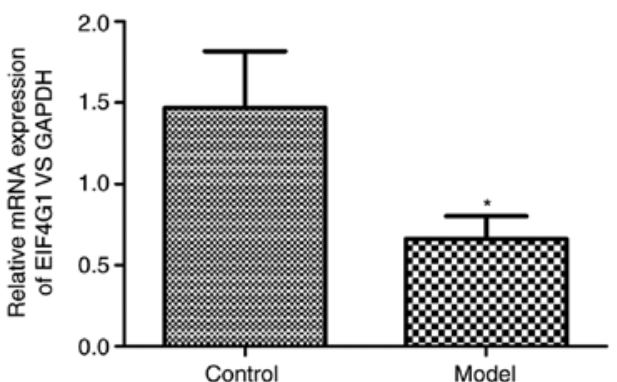

Figure 5. Relative expression levels of B4GALT1 and EIF4G1 between osteoarthritis samples and control samples. (A) Forest plots of the expression values of the key genes. Squares indicate the outcome estimates for the corresponding study and the size of the square indicates the weight of the corresponding study. Horizontal lines and figures in parentheses represent the 95\% CI. Diamonds indicate the pooled effect size with the corresponding 95\% CI. (B) Relative expression of B4GALT1 and EIF4G1 mRNA vs. GAPDH in synovial tissues of a rat model of OA vs. control. " $\mathrm{P}<0.05$; ${ }^{* *} \mathrm{P}<0.01$ vs. Control. Exp, expression; OR, odds ratio; CI, confidence interval; Pt, platform; PMID, PubMed unique identifier; B4GALT1, $\beta$-1,4-galactosyltransferase-1; EIF4G1, eukaryotic translation initiation factor $4 \gamma 1$. 
considered a key candidate gene associated with the development of OA.

EIF4G1 is a component of the multi-subunit protein complex EIF4F and limits the initiation phase of protein synthesis by stimulating cap-dependent translation (38). In a previous study, citrullinated EIF4G1 was identified as a novel auto-antigen in the pathogenesis of RA (39). As a paralog of EIF4G1, EIF4G2 is a key mediator of translation initiation and closely linked to mitosis and apoptosis (40). EIF4G2 was reported to be downregulated in OA cartilage and negatively correlated with microRNA-139, which inhibits chondrocyte proliferation and migration (41). It is therefore indicated that EIF4G1 may have an important role in OA. Of note, a recent study further confirmed that EIF4G1 was markedly changed in synovial membrane tissue affected by OA (42). Similarly, in the present study, EIF4G1 was identified as a key feature gene of OA and to be downregulated in OA samples compared with healthy controls.

Although B4GALT1 and EIF4G1 were verified to be downregulated by RT-qPCR in OA samples compared with healthy controls, the present study had certain limitations. However, the expression of B4GALT1 did not differ significantly between the OA and control samples as determined via the meta-analysis of five datasets. Of these, only dataset GSE1919 exhibited a different trend from the others, which was inconsistent with the result of RT-qPCR. This may be attributed to the small sample size of this dataset, resulting in significant data errors and large data fluctuations. Furthermore, RT-qPCR verification was only conducted using model rat samples, without OA patient samples. In the future, the synovial membrane samples from OA patients should be analyzed to verify the expression of these key genes. In addition, only the expression of two genes was verified using RT-qPCR, and thus, more key genes should be verified using RT-qPCR and western blot analyses. Furthermore, the interaction of these genes and their relevant biological functions and pathways will be explored in further studies.

In conclusion, the present study indicated that B4GALT1 and EIF4G1 were downregulated in synovial tissues affected by OA compared with those in healthy controls. B4GALT1 may be involved in the biological processes of OA, including positive regulation of developmental process, protein amino acid terminal glycosylation and protein amino acid terminal $\mathrm{N}$-glycosylation. These results may enhance the current understanding of the mechanisms underlying OA and provide novel targets for its treatment.

\section{Acknowledgements}

Not applicable.

\section{Funding}

No funding was received.

\section{Availability of data and materials}

The datasets used and/or analyzed during the current study are available from the corresponding author on reasonable request.

\section{Authors' contributions}

XS, HD and JM designed the study. LX and SY acquired the data. XS and HD performed the animal experiments. QH, XC and $\mathrm{WZ}$ analyzed the data. XS drafted the manuscript. JM revised the manuscript for important intellectual content. All authors read and approved the final manuscript.

\section{Ethics approval and consent to participate}

The present study was performed with the ethical approval of Honghui Hospital Affiliated to College of Medicine Xi'an Jiaotong University (Xi'an, China).

\section{Patient consent for publication}

Not applicable.

\section{Competing interests}

The authors declare that they have no competing interests.

\section{References}

1. Loeser RF, Goldring SR, Scanzello CR and Goldring MB: Osteoarthritis: A disease of the joint as an organ. Arthritis Rheum 64: 1697-1707, 2012.

2. Sagar DR, Ashraf S, Xu L, Burston JJ, Menhinick MR, Poulter CL, Bennett AJ, Walsh DA and Chapman V: Osteoprotegerin reduces the development of pain behaviour and joint pathology in a model of osteoarthritis. Ann Rheum Dis 73: 1558-1565, 2014.

3. Glynjones S, Palmer AJ, Agricola R, Price AJ, Vincent TL, Weinans H and Carr AJ: Osteoarthritis. Lancet 386: 376-387, 2015.

4. Vos T, Allen C, Arora M, Barber RM, Bhutta ZA, Brown A, Carter A, Casey DC, Charlson FJ, Chen AZ and Coggeshall M: Global, regional, and national incidence, prevalence, and years lived with disability for 310 diseases and injuries, 1990-2015: A systematic analysis for the global burden of disease study 2015 . Lancet 388: 1545-1602, 2016.

5. Hashimoto S, Ochs RL, Komiya S and Lotz M: Linkage of chondrocyte apoptosis and cartilage degradation in human osteoarthritis. Arthritis Rheum 41: 1632-1638, 1998.

6. Kaneko S, Satoh T, Chiba J, Ju C, Inoue K and Kagawa J: Interleukin-6 and interleukin-8 levels in serum and synovial fluid of patients with osteoarthritis. Cytokines Cell Mol Ther 6: 71-79, 2000.

7. Roach HI, Yamada N, Cheung KS, Tilley S, Clarke NM, Oreffo RO, Kokubun S and Bronner F: Association between the abnormal expression of matrix-degrading enzymes by human osteoarthritic chondrocytes and demethylation of specific $\mathrm{CpG}$ sites in the promoter regions. Arthritis Rheum 52: 3110-3124, 2005.

8. O'neill TW, Parkes MJ, Maricar N, Marjanovic EJ, Hodgson R, Gait AD, Cootes TF, Hutchinson CE and Felson DT: Synovial tissue volume: A treatment target in knee osteoarthritis (OA). Ann Rheum Dis 75: 84-90, 2016.

9. Hackinger S, Trajanoska K, Styrkarsdottir U, Zengini E, Steinberg J, Ritchie GRS, Hatzikotoulas K, Gilly A, Evangelou E, Kemp JP, et al: Evaluation of shared genetic aetiology between osteoarthritis and bone mineral density identifies SMAD3 as a novel osteoarthritis risk locus. Hum Mol Genet 26: 3850-3858, 2017.

10. Koike M, Nojiri H, Ozawa Y, Watanabe K, Muramatsu Y, Kaneko H, Morikawa D, Kobayashi K, Saita Y, Sasho T, et al: Mechanical overloading causes mitochondrial superoxide and SOD2 imbalance in chondrocytes resulting in cartilage degeneration. Sci Rep 5: 11722, 2015.

11. Muñoz-Valle JF, Oregón-Romero E, Rangel-Villalobos H, Martínez-Bonilla GE, Castañeda-Saucedo E, Salgado-Goytia L, Leyva-Vázquez MA, Illades-Aguiar B, Alarcón-Romero Ldel C, Espinoza-Rojo $M$ and Parra-Rojas I: High expression of TNF alpha is associated with- 308 and- 238 TNF alpha polymorphisms in knee osteoarthritis. Clin Exp Med 14: 61-67, 2014. 
12. Jiang Y, Hu C, Yu S, Yan J, Peng H, Ouyang HW and Tuan RS Cartilage stem/progenitor cells are activated in osteoarthritis via interleukin-1 $\beta /$ nerve growth factor signaling. Arthritis Res Ther 17: 327, 2015.

13. Vuolteenaho K, Koskinen-Kolasa A, Laavola M, Nieminen R, Moilanen T and Moilanen E: High synovial fluid interleukin-6 levels are associated with increased matrix metalloproteinase levels and severe radiographic changes in osteoarthritis patients. Osteoarthritis Cartilage 25 (Suppl 1): S92-S93, 2017.

14. Guns LA, Kvasnytsia M, Kerckhofs G, Vandooren J, Martens E, Opdenakker G, Lories RJ and Cailotto F: Suramin protects against osteoarthritis by increasing tissue inhibitor of matrix metalloproteinase-3 and glycosaminoglycans in the articular cartilage. Osteoarthritis Cartilage 25: S145-S146, 2017.

15. van Helvoort EM, Popov-Celeketic J, Coeleveld K, Tryfonidou MA, Lafeber FP and Mastbergen SC: Effects of the human IL4-10 fusion protein in the canine groove model of osteoarthritis. Osteoarthritis Cartilage 25: S434, 2017.

16. Ratnayake M, Plöger F, Santibanez-Koref M and Loughlin J: Human chondrocytes respond discordantly to the protein encoded by the osteoarthritis susceptibility gene GDF5. PLoS One 9: e86590, 2014.

17. Nepple JJ, Thomason KM, An TW, Harris-Hayes M and Clohisy JC: What is the utility of biomarkers for assessing the pathophysiology of hip osteoarthritis? A systematic review. Clin Orthop Relat Res 473: 1683-1701, 2015.

18. Sliz E, Taipale M, Welling M, Skarp S, Alaraudanjoki V, Ignatius J, Ruddock L, Nissi R and Männikkö M: TUFT1, a novel candidate gene for metatarsophalangeal osteoarthritis, plays a role in chondrogenesis on a calcium-related pathway. PLoS One 12: e0175474, 2017.

19. Carvalho BS and Irizarry RA: A framework for oligonucleotide microarray preprocessing. Bioinformatics 26: 2363-2367, 2010

20. Troyanskaya O, Cantor M, Sherlock G, Brown P, Hastie T, Tibshirani R, Botstein D and Altman RB: Missing value estimation methods for DNA microarrays. Bioinformatics 17: 520-525, 2001.

21. Rao Y, Lee Y, Jarjoura D, Ruppert AS, Liu CG, Hsu JC and Hagan JP: A comparison of normalization techniques for microRNA microarray data. Stat Appl Genet Mol Biol 7: Article22, 2008.

22. Kang DD, Sibille E, Kaminski $N$ and Tseng GC: MetaQC: Objective quality control and inclusion/exclusion criteria for genomic meta-analysis. Nucleic Acids Res 40: e15, 2011.

23. Demšar U, Harris $P$, Brunsdon $C$, Fotheringham AS and McLoone S: Principal component analysis on spatial data: An overview. Ann Assoc Am Geogr 103: 106-128, 2013.

24. Wang X, Kang DD, Shen K, Song C, Lu S, Chang LC, Liao SG, Huo Z, Tang S, Ding Y, et al: An R package suite for microarray meta-analysis in quality control, differentially expressed gene analysis and pathway enrichment detection. Bioinformatics 28 : 2534-2536, 2012.

25. Szklarczyk D, Franceschini A, Wyder S, Forslund K, Heller D, Huerta-Cepas J, Simonovic M, Roth A, Santos A, Tsafou KP, et al: STRING v10: Protein-protein interaction networks, integrated over the tree of life. Nucleic Acids Res 43 (Database Issue) D447-D452, 2015.

26. Jeong H, Mason SP, Barabási AL and Oltvai ZN: Lethality and centrality in protein networks. Nature 411: 41-42, 2001.

27. Alizadeh E, Lyons S, Castle J and Prasad A: Measuring systematic changes in invasive cancer cell shape using Zernike moments. Integr Biol (Camb) 8: 1183-1193, 2016.
28. Huang da W, Sherman BT and Lempicki RA: Systematic and integrative analysis of large gene lists using DAVID bioinformatics resources. Nat Protoc 4: 44-57, 2009.

29. Huang da W, Sherman BT and Lempicki RA: Bioinformatics enrichment tools: Paths toward the comprehensive functional analysis of large gene lists. Nucleic Acids Res 37: 1-13, 2009.

30. Hayami T, Pickarski M, Zhuo Y, Wesolowski GA, Rodan GA and Duong LT: Characterization of articular cartilage and subchondral bone changes in the rat anterior cruciate ligament transection and meniscectomized models of osteoarthritis. Bone 38: 234-243, 2006.

31. Livak KJ and Schmittgen TD: Analysis of relative gene expression data using real-time quantitative PCR and the 2(-Delta Delta C(T)) method. Methods 25: 402-408, 2001

32. Réka A: Scale-free networks in cell biology. J Cell Sci 118: 4947-4957, 2005

33. Lei X, Wu FX, Tian J and Zhao J: ABC and IFC: Modules detection method for PPI network. Biomed Res Int 2014: 968173, 2014

34. Qasba PK, Ramakrishnan B and Boeggeman E: Structure and function of beta-1,4-galactosyltransferase. Curr Drug Targets 9: 292-309, 2008

35. Al-Obaide MA, Alobydi H, Abdelsalam AG, Zhang R and Srivenugopal KS: Multifaceted roles of 5'-regulatory region of the cancer associated gene B4GALT1 and its comparison with the gene family. Int J Oncoly 47: 1393-1404, 2015.

36. Liu W, Cui Z, Wang Y, Zhu X, Fan J, Bao G, Qiu J and $\mathrm{Xu} \mathrm{D}$ : Elevated expression of $\beta 1,4$-galactosyltransferase-I in cartilage and synovial tissue of patients with osteoarthritis. Inflammation 35: 647-655, 2012.

37. Sun Y, Franklin AM, Mauerhan DR and Hanley EN: biological effects of phosphocitrate on osteoarthritic articular chondrocytes. Open Rheumatol J 11: 62-74, 2017.

38. Park EH, Walker SE, Lee JM, Rothenburg S, Lorsch JR and Hinnebusch AG: Multiple elements in the eIF4G1 N-terminus promote assembly of eIF4G1 •PABP mRNPs in vivo. EMBO J 30: 302-316, 2011.

39. Okazaki Y, Suzuki A, Sawada T, Ohtake-Yamanaka M, Inoue T, Hasebe T, Yamada R and Yamamoto K: Identification of citrullinated eukaryotic translation initiation factor $4 \mathrm{G} 1$ as novel autoantigen in rheumatoid arthritis. Biochem Biophys Res Commun 341: 94-100, 2006.

40. Coldwell MJ, Cowan JL, Vlasak M, Mead A, Willett M, Perry LS and Morley SJ: Phosphorylation of eIF4GII and 4E-BP1 in response to nocodazole treatment: A reappraisal of translation initiation during mitosis. Cell Cycle 12: 3615-3628, 2013.

41. Hu W, Zhang W, Li F, Guo F and Chen A: miR-139 is up-regulated in osteoarthritis and inhibits chondrocyte proliferation and migration possibly via suppressing EIF4G2 and IGF1R. Biochem Biophys Res Commun 474: 296-302, 2016.

42. Zhang X, Yuan Z and Cui S: Identifying candidate genes involved in osteoarthritis through bioinformatics analysis. Clin Exp Rheumatol 34: 282-290, 2016.

This work is licensed under a Creative Commons Attribution-NonCommercial-NoDerivatives 4.0 International (CC BY-NC-ND 4.0) License. 